\title{
Gobierno de datos, un potenciador de los sistemas de gestión de calidad $^{*}$
}

Data government, a potential of quality management systems

Administração de dados, um intensificador de sistemas de gestão de qualidade

Juan Dario Portilla Romero**

Ministerio de Hacienda y Crédito Público

*Artículo de resultado de investigación. DOI: http://dx.doi.org/10.15332/s2145-1389.2017.0002.10

** Magíster en Calidad y Gestión Integral. Especialista en Gerencia de Proyectos de Ingeniería. Ingeniero Industrial. Consultor Sistema Único de Gestión en el Ministerio de Hacienda y Crédito Público. Correo electrónico: juandario0303@gmail.com. 


\section{RESUMEN}

La gestión de datos es una herramienta eficaz para el desarrollo de los componentes de un sistema de gestión en las organizaciones. Además, permite la disposición de las fuerzas productivas en busca de generar un mayor valor y la distribución de los beneficios que trae la globalización para superar las múltiples desigualdades en cumplimiento del objeto social de Positiva Compañía de Seguros S. A., como única aseguradora estatal del mercado de personas. Atendiendo a ello, la investigación tuvo como objetivo determinar los efectos de la implementación de un programa de Gobierno de Datos (GD) integrado al Sistema de Gestión de la Calidad en Positiva. Se indagó de forma cualitativa por la percepción de los usuarios de los datos acerca de la implementación del GD y se identificaron los impactos generados tras su implementación en el 2012. Como resultado se identificaron los aportes del GD a los componentes de requisitos generales, responsabilidad de la dirección y medición, análisis y mejora de la norma ISO 9001. Se concluyó que la implementación del GD fue buena según los usuarios de los datos y se identificaron efectos importantes que aportan a disminuir la siniestralidad y lograr la meta institucional.

Palabras clave: sistemas de gestión, calidad, gobierno de datos.

\section{ABSTRACT \\ Data management is an effective tool for the develop- ment of the components of a management system in organizations. In addition, it allows the disposition of the productive forces in search of generating a greater value and the distribution of the benefits that brings the globalization to overcome the multiple inequali- ties in fulfillment of the social object of Positive In- surance Company S.A., like unique insurer state of the}

market of people. Accordingly, the research aimed to determine the effects of the implementation of a Data Governance (DG) program integrated into the Quality Management System in Positiva. Qualitative inquiries were made by the users' perception of the data about the implementation of the DG and the impacts generated after its implementation in 2012 were identified. As a result, DG's contributions to the general requirements components, the management and measurement, analysis and improvement of ISO 9001. It was concluded that the implementation of the GD was good according to the users of the data and important effects were identified that contribute to decrease the accidents and achieve the institutional goal.

Keywords: management system, quality, data governance.

\section{RESUMO}

A pesquisa visa determinar os efeitos da implementação de um programa de Administração de Dados integrados ao Sistema de Gestão de Qualidade em Positiva Companhia de Seguros S.A., para ele deve-se determinar as contribuições do GD ao SGC, conhecer a percepção dos usuários dos dados sobre a implementação do GD e fazer uma análise, identificando os impactos gerados após a implementação da administração de dados em 2012. Esta pesquisa é baseada no layout das forças produtivas visando gerar maior valor, a distribuição dos benefícios que a globalização traz para superar as múltiplas desigualdades em cumprimento do objeto social de Positiva como única seguradora estatal do mercado de pessoas, buscando satisfazer às necessidades dos clientes executando processos suportados em um sistema de governo relacionados aos dados e informações. Esta pesquisa tem uma abordagem positivista e utilizou um método quantitativo, como resultado identificaram-se às contribuições do 
GD aos componentes dos requisitos gerais, responsabilidade da direção e medidas, análise e melhoria da norma ISO 9001, se concluiu que a implementação do GD foi boa de acordo com os usuários dos dados e se identificou efeitos importantes que contribuem para reduzir acidentes e alcançar o objetivo institucional. A importância da pesquisa estabelece que 0 GD é uma ferramenta eficaz para o desenvolvimento dos componentes de um SGC em uma companhia de seguros que gera valor para seus segurados.

Palavras-chave: sistemas de gestão, qualidade, administração de dados.

\section{INTRODUCCIÓN}

El gobierno de datos (GD) es una práctica relativamente nueva, según el análisis de antecedentes internacionales se encontró que los problemas relacionados con los datos e información inicialmente se abordaban desde la perspectiva del almacenamiento pensando que el problema radicaba en la capacidad para salvaguardar la información. Cuando se solucionó el tema de la capacidad de almacenaje, siguieron los problemas de administración, en donde se optó por gestionar a su vez el contenido de la información. De esta preocupación surgió la necesidad de construir estrategias, políticas 0 estructuras que permitan gestionar los contenidos de la información (Thomas, s. f.; Thomas, 2009). La gestión de la información garantizará el intercambio eficiente de estos datos (Buchmann, 2017).

Otro inconveniente para las organizaciones ha sido la seguridad de la información. De acuerdo con lo expresado por Mansfield-Devine (2017), es a menudo una reflexión tardía en la planificación y el gasto de una organización en la planeación de la información tecnológica y rara vez recibe la atención de alto nivel que necesita y merece.
Atendiendo al anterior contexto, las empresas se han vuelto más sofisticadas en su uso de los datos que impulsa nuevas demandas que requieren diferentes formas de combinar, manipular, almacenar y presentar información. Por ello, se han desarrollado programas para la gestión efectiva de datos a través de nubes, lo cual incluye el punto de vista tanto del consumidor como del proveedor (Al-Ruithe, Benkhelifa y Hameed, 2016).

Otro de los usos de la gestión de los datos ha sido utilizar datos importantes para promover la sostenibilidad (Seele, 2017; Bibri y Krogstie, 2017) y reducir el daño (entre ellos el ambiental) ocasionado por las organizaciones. Para esto se creó el concepto de "control predictivo de sostenibilidad", que corresponde al uso de técnicas analíticas para identificar temas de deliberación, supervisión e intervención con el objetivo de prevenir futuros daños ambientales, sociales y de gobernanza, resolviendo escándalos pasados e identificando a actores/corporaciones potenciales de actividades insostenibles y sus partes interesadas en un futuro próximo (Seele, 2017).

Para la investigación se determinó como objeto de estudio Positiva Compañía de Seguros S. A., la cual en el año 2012 implementó un programa de gobierno de datos con el fin de dar solución a problemas derivados de brechas encontradas en la administración de los datos e información; es importante precisar que a la compañía desde el mismo momento de su creación en el año 2008, siempre le ha interesado contar con un sistema integrado de gestión, para este caso el sistema está compuesto por las normas ISO 9001:2008, ISO 14001:2004, OSHAS 18001:2007, NTCGP 100:2009 y MECI, estas últimas se incluyen en el sistema por ser Positiva una empresa de carácter estatal; además, la Oficina de Estrategia y Desarrollo en su calidad de encargada de la implementación y mantenimiento del sistema integrado, tiene como política que cualquier 
sistema, modelo o directriz relacionada con gestión corporativa debe estar alineado con las políticas transversales del sistema integrado de gestión. El programa de gobierno de datos actualmente se encuentra integrado con los sistemas implementados. Para efectos de la investigación solamente se tendrá en cuenta el Sistema de Gestión de la Calidad basado en la norma ISO 9001:2008.

Los motivadores de la investigación fueron en un principio, el desconocimiento por parte de los responsables de gestionar los datos en la compañía, de los efectos que tuvo la implementación del gobierno de datos (GD) en Positiva. Además, la entidad no cuenta con alguna dimensión de los impactos de la misma, ni tampoco se tiene una idea acerca de cómo el gobierno de datos ha aportado al desempeño del Sistema de Gestión de la Calidad implementado en la compañía y la forma en que esta articulación está ayudando a cumplir la meta corporativa de tener el índice combinado menor a $100 \%$ al año 2018.

Todos los análisis de esta investigación parten del supuesto que, Positiva a través de la incorporación del gobierno de datos al Sistema de Gestión de la Calidad, logra mejorar el desempeño de la compañía generando beneficios cualitativos y cuantitativos para esta, ya que esta afirmación resume lo que la alta dirección espera después de haber realizado la implementación del programa en el año 2012.

Para alcanzar el objetivo general de la investigación, se considera que se debe empezar por identificar la forma en que los componentes propios del programa de gobierno de datos aportan a los elementos de un Sistema de Gestión de la Calidad y mostrar de qué forma se complementan los dos sistemas a través del análisis de los aportes y la descripción de los resultados puestos en marcha en la operación; seguido del diseño y construcción de un instrumento de recolección de información que permita medir la percepción de los usuarios de los datos e información en la compañía buscando fortalezas y debilidades dentro del proceso implementado en el año 2012; para finalizar, se identifican los efectos de la implementación, a partir de situaciones que han tenido intervención de los componentes de gobierno de datos y se realiza una cuantificación monetaria de resultados de estos efectos encontrados; por último, una vez se concreten resultados, se procede a realizar los respectivos análisis de los tres objetivos específicos y se describen cuáles son los impactos en la operación de la compañía. Estos resultados serán comunicados a la compañía bajo el entendido de que hasta el momento no se ha realizado un análisis posterior a la implementación del GD en el año 2012 y la alta dirección estaría muy interesada en conocer por primera vez los impactos posteriores a la implementación de uno de los proyectos que aprobaron en años anteriores.

\section{METODOLOGÍA}

La presente investigación buscó solucionar el problema a través de la presentación de resultados que reflejen los efectos de la implementación del gobierno de datos en Positiva articulado con el Sistema de Gestión de la Calidad. En el contexto teórico, la investigación es de carácter aplicado, ya que utiliza teorías que ya han sido probadas anteriormente, como lo es el modelo de gobierno de datos y modelo de gestión de la calidad (ICONTEC, 2008), los cuales tiene relación con problemas reales en organizaciones, para este caso puntual, para Positiva Compañía de Seguros. Lo anterior permite ir desde el planteamiento de un problema a buscarle una solución, de acuerdo con una situación actual; para ello se utilizará un desarrollo metodológico a través de la observación directa de los efectos del gobierno de datos analizando las situaciones, identificando posibles efectos, basados en información histórica, indicadores de gestión, revisiones por la dirección 
e históricos de PQR; todo esto con el fin de llegar a conclusiones a partir de los hallazgos de este proceso.

A su vez, la investigación se fundamenta epistemológicamente en el paradigma positivista, ya que busca probar la hipótesis presentada, a través de la recolección de datos, transformar la información para análisis estadístico y con base en la medición numérica, establecer patrones de comportamiento y probar teorías, es decir, se utilizará un enfoque cuantitativo (Hernández, Fernández y Baptista, 2014). De acuerdo el alcance de esta investigación, esta es de carácter explicativa, porque a partir de la caracterización de la situación inicial en el año 2012, en Positiva cuando se implementó el gobierno de datos, se concluye mostrando cuáles son los efectos de la implementación en la operación normal de la compañía.

En esta investigación se utilizará un método inductivo porque se parte de la observación de fenómenos particulares de Positiva S. A., para llegar a generalizaciones o conclusiones que se puedan aplicar en organizaciones similares de forma general por medio del razonamiento lógico y análisis estadístico a partir de varias suposiciones. Con base en ello y haciendo uso de los instrumentos y técnicas de investigación correspondientes, se elabora un diagnóstico de la organización que haga posible identificar las características actuales de su comportamiento respecto del manejo de la información y de los datos, y las respuestas de sus grupos de interés.

Una vez definido el diseño metodológico, se procede a construir los instrumentos de recolección de información y se seleccionan las variables a analizar. Como primera medida, está la encuesta de percepción donde por cada uno de los componentes del gobierno de datos, se definen 10 puntos en busca de hacer una validación de contenido, para que los expertos califiquen según su criterio y experiencia la pertinencia de las preguntas que componen el instrumento a aplicar. Posteriormente, se tabulan los resultados y los puntos con concepto favorable para la aplicación harán parte del instrumento; 10 anterior se realiza para garantizar que el instrumento es coherente con los objetivos de la investigación y cumplen con el rigor técnico para que sean aplicados en el instrumento.

Surtido el proceso anterior, se procedió a construir el instrumento de medición compuesto por 25 preguntas, este instrumento se aplicará a la muestra seleccionada de los usuarios de los datos en Positiva. La compañía en total cuenta con 749 trabajadores, de los cuales 407 se encuentran en la casa matriz, siendo esta la población a analizar; el diseño de la muestra arroja que para que esta sea representativa se deben aplicar 87 encuestas, la muestra está constituida por un grupo que tiene estrecha relación con el uso de los datos y tiene unas características particulares: en primer lugar, se encuentran los gerentes públicos, para efectos del sistema integrado de gestión ellos tienen el rol de líderes de proceso teniendo como responsabilidad tomar decisiones, en total se cuenta con 38 gerentes públicos; en segundo lugar, se encuentran los funcionarios que tienen un rol de líder SIG, este rol lo tienen los funcionarios que llevan el control de las métricas para cada uno de los procesos, en total son 41 líderes SIG, uno por cada proceso; por último y para completar la población a analizar, se encuentran los funcionarios que tienen el rol de líderes BSC, los cuales pertenecen a cada una de las vicepresidencias de la compañía, estos se encargan de reportar los indicadores correspondientes al balanced score card, en total son 8 líderes.

Para determinar los aportes del gobierno de datos (GD) al Sistema de Gestión de la Calidad y realizar el análisis de los impactos en el desempeño institucional después de su implementación, se aplica la técnica de recolección de datos a través de la observación, la cual está definida como uno de los instrumentos más prác- 
ticos usados en la investigación científica. Esta técnica permite percibir directamente los hechos de la realidad objetiva, acompañada del levantamiento de información de fuentes primarias de información y concluir conjugando estos dos elementos.
Teniendo en cuenta lo anterior, el investigador propone determinar los efectos de la implementación de un programa de gobierno de datos (GD) articulado con el Sistema de Gestión de Calidad en Positiva Compañía de Seguros S. A.

Tabla 1. Aportes de GD al Sistema de Gestión de la Calidad ISO 9001:2008.

\section{COMPONENTES NORMA ISO 9001:2008}

Capítulo 3 - Términos y definiciones

4.1 Definiciones ISO 9000

Capítulo 4 - Requisitos del sistema

4.1 Requisitos generales

4.2 Requisitos documentales

Capítulo 5 - Responsabilidades de la Dirección

5.1 Compromiso de la Dirección

5.2 Enfoque al cliente

5.3 Política de calidad

5.4 Planificación

5.5 Responsabilidad, autoridad y comunicación

5.6 Revisión por la Dirección

Capítulo 6 - Gestión de recursos

6.1 Provisión de recursos

6.2 Recursos humanos

6.3 Infraestructura

6.4 Ambiente de trabajo

Capítulo 7 - Realización del producto

7.1 Planificación de la realización del producto

7.2 Procesos relacionados con el cliente

7.3 Diseño y desarrollo

7.4 Compras

7.5 Producción y prestación del servicio

7.6 Control dispositivos de seguimiento y de medición (N.A.)

Capítulo 8 - Medición, análisis y mejora

8.1 Generalidades

8.2 Seguimiento y medición

8.3 Control de los productos no conformes

8.4 Análisis de los datos

8.5 Mejora

\section{Componentes de Gobierno de Datos}

$\begin{array}{llllllllll}1 & 2 & 3 & 4 & 5 & 6 & 7 & 8 & 9 & 10\end{array}$
X \begin{tabular}{|l|l|l|l|l|l|l|l|l|}
\hline $\mathrm{X}$ & $\mathrm{X}$ & $\mathrm{X}$ & $\mathrm{X}$ & $\mathrm{X}$ & $\mathrm{X}$ & & $\mathrm{X}$ & $\mathrm{X}$ \\
\hline & & $\mathrm{X}$ & $\mathrm{X}$ & & & & $\mathrm{X}$ & \\
\hline
\end{tabular}

\begin{tabular}{|l|l|l|l|l|l|l|l|l|l|}
\hline $\mathrm{X}$ & & & $\mathrm{X}$ & $\mathrm{X}$ & & $\mathrm{X}$ & & & \\
\hline & & $\mathrm{X}$ & & & & & & & $\mathrm{X}$ \\
\hline $\mathrm{X}$ & $\mathrm{X}$ & & $\mathrm{X}$ & & $\mathrm{X}$ & & $\mathrm{X}$ & \\
\hline $\mathrm{X}$ & & $\mathrm{X}$ & & $\mathrm{X}$ & & $\mathrm{X}$ & $\mathrm{X}$ & & \\
\hline & & & $\mathrm{X}$ & & & & & \\
\hline $\mathrm{X}$ & $\mathrm{X}$ & & & $\mathrm{X}$ & $\mathrm{X}$ & & & & \\
\hline
\end{tabular}

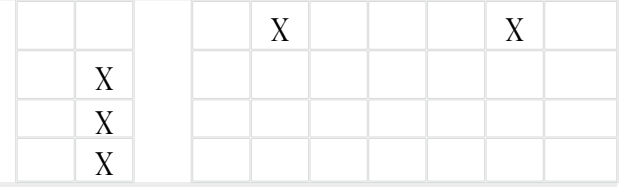


Según lo indica la tabla anterior, el gobierno de datos apoya en gran medida el desarrollo de cada uno de los elementos del Sistema de Gestión de la Calidad, esta calificación se realizó teniendo en cuenta que el componente de gobierno de datos aporte de alguna forma algún elemento al capítulo analizado, resultando que los capítulos: 4. Requisitos del sistema, con el $50 \%$ de aportación; 5. Responsabilidades de la Dirección, con el $38 \%$; y, 8. Medición, análisis y mejora con el 50 $\%$; son los más beneficiados con la implementación del programa. Una vez se identificaron cuáles fueron los capítulos de la norma que se beneficiaron más, se procedió a definir cuáles fueron los aportes en cada uno de ellos, los resultados fueron los siguientes:

AP01. Incorporación de proceso de gobierno de datos en el SIG. En el año 2013 se creó formalmente el proceso de gobierno de datos en la compañía, este proceso está a cargo de la Oficina de Estrategia y Desarrollo y tiene como objetivo establecer las herramientas necesarias que permitan contar con información precisa, segura y oportuna para clientes, empleados y demás terceros que requieran información de la compañía. Este proceso cuenta con un líder de proceso a cargo del jefe de la Oficina de Estrategia y Desarrollo y un líder SIG a cargo de un profesional especializado; a su vez se desarrollaron procedimientos de: a) protección de datos personales, b) calidad de datos, c) arquitectura de datos y, d) gestión de metadata; la gestión de los datos se aplica cuando la compañía identifica problemas relacionados con los datos e información y se brinda apoyo a las iniciativas o proyectos que estén relacionados con datos en la compañía, así mismo se intervienen con la aplicación de los componentes propios del GD.

AP02. Incorporación del proceso de inteligencia de negocios y creación de bodega de datos como repositorio oficial de la compañía. El proceso creado desde el año 2008 y la bodega conformada en el año 2013; estos dos elementos son indispensables para la compa- ñía, el análisis de la información a través del monitoreo constante y el análisis de los datos observados en las variables claves del negocio para proveer información relevante que sirva de soporte para la toma de decisiones acertadas y oportunas; adicionalmente con el propósito de buscar la consistencia, integración, unicidad de los datos y el apalancamiento tecnológico en modelos analíticos, la Oficina de Estrategia y Desarrollo y la Gerencia Informática unen esfuerzos para crear la bodega de datos como repositorio oficial ante la necesidad de contar con una fuente de información unificada en la compañía; para que la información fuera fácil de consultar y entender se desarrollaron en la bodega de datos, varios modelos analíticos de siniestralidad, afiliaciones ARL, suscripciones vida, finanzas y accidentalidad, a través de reportes estandarizados. Como resultado de esta implementación la bodega de datos corporativa se convierte en el repositorio oficial de los datos analíticos, buscando garantizar la consistencia e integridad de estos y generando credibilidad por parte de los usuarios de la información.

AP03. Generación de inventario de flujos de información. En el año 2012 se realizó el levantamiento de los flujos de información en el cual se describen los datos e información que se manejan entre los procesos, bases de datos utilizadas, almacenes de datos o las entidades externas relacionadas con la información. La intervención de los componentes del GD en este aporte, se evidencia a través de la definición de los roles y responsables de la información, siendo un complemento a los flujogramas de los procesos, lo cual redunda en un mejor entendimiento de las interacciones de actividades, personas e información.

AP04. Apoyo para el cumplimiento de la política de calidad, a través de la incorporación de mejores prácticas en la gestión de las métricas de procesos. Positiva cuenta con una política integral donde se tienen en cuenta cada uno de los sistemas de gestión que se 
encuentran implementados, estos se llaman principios fundamentales, siendo componentes: el Sistema de Gestión de la Calidad ISO 9001:2008, gestión ambiental ISO 14001, gestión de seguridad y salud en el trabajo, sistema de gestión de continuidad del negocio y modelo de sostenibilidad corporativa; dentro de la política, el principio fundamental en la política integral que se relaciona con el Sistema de Gestión de la Calidad es "servicio de excelencia para los clientes y asegurados", donde el sistema busca garantizar la eficiencia y eficacia que la operación de la compañía, esto no se puede lograr si no se pueden controlar los procesos, si no existe información confiable para la toma de decisiones que permitan tomar acciones para mejorar la satisfacción de los clientes.

Se analizaron 393 indicadores de los procesos desde el año 2012 y hasta el 2015, identificando cuáles son más sensibles a la calidad de los datos en lo referente a completitud, integridad y confiabilidad, siendo calificados según la criticidad por cada uno de estos atributos de calidad de datos. Como resultado del análisis se demuestra, a través de los 4 años, que se presenta una mejora al incrementarse en promedio 17 indicadores que tienen gran influencia del GD, esta tendencia muestra que a través de los años se ha ido consolidando la cultura de formulación de indicadores que tengan en cuenta los atributos de completitud, integridad y confiabilidad, brindando una herramienta que apoya los procesos de toma de decisiones.

AP05. Construcción de metadata corporativa. Positiva en el año 2012 no contaba con una forma efectiva de comunicarse al tener en cada área de la compañía un lenguaje distinto, el cual es importante unificar para evitar confusiones. La definición de metadata para la compañía tiene como propósito la existencia de un lenguaje común que facilite el análisis, posibilite la comprensión, refine las consultas de datos y clarifique la comunicación. A través de reuniones con todos los procesos, se levantaron metadatos de un total de 7500 términos, siendo utilizados en varios escenarios donde confluyen varias áreas, que sean los mismos y esto facilite el entendimiento entre los equipos multifuncionales.

P06. Esquema de Control de Análisis de Métricas Integrado CAMI y herramienta para Medición de Acuerdos de Gestión MAG. Contar con un mecanismo de control de su gestión unificado el cual centralice la información de los indicadores de gestión de la compañía fue una necesidad en el año 2012, esto debido a problemas relacionados con duplicidad de información, acceso y administración, con el fin de dar solución a esto se aplicaron los componentes del gobierno de datos. La Oficina de Estrategia y Desarrollo diseñó una herramienta cuya finalidad será la de contar con un gobierno mucho más eficiente de la información de gestión de la compañía, proporcionando una fuente única y confiable, donde se facilite la consulta, reporte y análisis para indicadores estratégicos y de procesos, también se construyeron reglas de negocio para la administración de la información contemplando roles y responsabilidades en todos los niveles de la organización.

Como complemento, se realiza el diseño y desarrollo de otra herramienta para la medición del desempeño de los funcionarios a través de los acuerdos de gestión de la compañía. Esta herramienta busca aprovechar la información estructurada almacenada en la base de datos del CAMI para que haga parte de los acuerdos de gestión de la compañía, siendo un apoyo importante para el desarrollo del talento humano, permitiendo tener un elemento cuantitativo directamente relacionado con los resultados de la compañía en la evaluación del desempeño personal. El uso y apropiación de esta herramienta se ha ido mejorando y poco a poco se han ido incorporando elementos importantes, como los análisis hecho-causa-acción para cada análisis de indicadores, orientando al sistema y a la mejora continua. 
AP07. Implementación de esquema de medición organizacional a los niveles estratégico, táctico y operativo. Apoyándose en la herramienta MAG, la compañía busca llevar el control del desempeño de la estrategia corporativa en todos sus niveles, buscando involucrar a los trabajadores de Positiva con el desempeño institucional y crear sentido de pertenencia con la compañía al atar sus evaluaciones de desempeño, con los resultados de la medición de los acuerdos de gestión. Se formulan planes de acción con compromisos de mejora personales, los cuales buscan que el desempeño de los colaboradores mantenga altos estándares. De la misma forma que se implementó el CAMI, se fijaron políticas, roles y responsabilidades, todas orientadas a la gobernabilidad de la información generada; en total se gestionan 749 acuerdos para la vigencia 2015, como beneficio para los trabajadores de Positiva dentro del pacto colectivo concertado entre la administración y los trabajadores, se determinó que los colaboradores que superen el $85 \%$ en su evaluación del desempeño tiene derecho al $15 \%$ de su salario; para el año 2015 se pagaron alrededor de $\$ 645^{\prime} 362.788$ en bonificaciones.

En desarrollo del objetivo de conocer la percepción de los usuarios de los datos en Positiva con relación a la implementación del gobierno de datos, se construyó una encuesta tipo Likert, la cual se aplicó a los usuarios de los datos en la compañía; la encuesta se remitió a 87 individuos seleccionados como muestra y en total se obtuvieron 81 respuestas, lo que representa el 93,1\% de diligenciamiento. El índice de percepción arrojado por la encuesta es de 3,82, lo cual indica que en general los usuarios de los datos e información en Positiva tienen una buena percepción frente a la implementación del GD.

Dentro de las afirmaciones que obtuvieron mayor calificación, estuvieron: 1) El proceso de gobierno de datos (GD) se encuentra formalmente establecido en el Sistema Integrado de Gestión (SIG) de la compañía.
El $8 \%$ de los encuestados dieron calificación positiva a esta afirmación, lo que demuestra que los procesos de comunicación desde la implementación en el año 2012 han sido efectivos y han generado recordación en los niveles estratégico, táctico y operativo. 2) $\mathrm{La}$ información generada al interior de mi proceso es confiable, precisa y sirve para la adecuada toma de decisiones por parte de los líderes de procesos. Con $88 \%$ de las respuestas positivas, se evidencia que se ha generado cierta confianza en la información que se genera al interior de los procesos, esto gracias a los resultados obtenidos después de la implementación. 3) En el proceso al cual usted pertenece se tienen identificados quiénes son los que suministran los datos y sus respectivas líneas de reporte con los demás procesos. Con $88 \%$ de respuestas positivas, La construcción de los flujos de información y el inventario de activos de información ha ayudado a que los procesos tengan claro en qué parte de la cadena de valor están, además de mejorar el entendimiento de la trazabilidad. 4) Usted identifica claramente un área en la compañía que se encargue de gestionar el GD. Con $84 \%$ de respuesta positivas, los encuestados reconocen que hay un área que centraliza la información y gestiona el GD, en este caso es la Oficina de Estrategia y Desarrollo. 5) La gestión del riesgo del proceso al cual usted pertenece incluye controles para la mitigación de riesgos relacionados con los datos e información. Con $85 \%$ de calificaciones positivas, los encuestados opinan que cuentan con los controles necesarios para gestionar los riesgos, esto se soporta también en la proliferación de indicadores que tengan sensibilidad ante la calidad de la información. 6) En el proceso al cual usted pertenece ha realizado acercamientos para la unificación de los términos utilizados con las diferentes áreas y procesos con los que se relacionan. Con 81 $\%$ de respuestas positivas, demuestra el compromiso que tiene los procesos al realizar mesas transversales para construir su metadata. 
Las afirmaciones con menor calificación fueron: 1) Existen indicadores definidos para administrar la calidad de los datos o información a nivel organizacional. Con el $39 \%$ de respuestas negativas, los procesos cuentan con indicadores relacionados con el gobierno de datos, pero no con un indicador global o un agregado de varias mediciones relacionadas con la calidad de los datos. 2) En el proceso al cual usted pertenece tiene definidos indicadores y metas relacionados con la gestión de los datos o información. Con $34 \%$ de respuesta negativas, se puede inferir que la respuesta es subjetiva al tener, del $66 \%$ de las respuestas negativas el $55 \%$ corresponde a respuestas: ni de acuerdo ni en desacuerdo, en la mayoría de los casos se responde de esta manera cuando hay desconocimiento del tema que se está preguntando. 3) Se conoce el grado de satisfacción de las partes interesadas después de que son solucionadas sus necesidades relacionadas con los datos. Con $46 \%$ de respuestas negativas, una de las grandes debilidades del GD es que los procesos no miden el grado de satisfacción de los datos e información que se entrega a sus partes interesadas. 4) El objetivo y alcance del proceso de GD ha sido comunicado y es comprendido por todos los niveles de la compañía. Con $44 \%$ de respuesta negativas, se denota que los encuestados desconocen el objetivo y el alcance del proceso haciendo esto entender que se debe hacer un trabajo más directo en el momento de capacitar y dar a conocer el proceso a la compañía.

Por último, se muestran los resultados de los análisis de efectos en la operación de la compañía después de la implementación del GD.

Efecto 1. Reducción en los niveles de exposición al riesgo de la compañía por suscripción inadecuada del riesgo en las empresas.

- Tipo de efecto: Ahorro en operación

- Cuantificación: \$3.485’916.000.00 por año
Este efecto se centra en el proceso de entrada de un cliente a la compañía, en específico en el momento de la afiliación donde se realiza la suscripción del riesgo y se tratan de realizar todas las actividades necesarias para que las nuevas empresas afiliadas, ingresen a la compañía en condiciones justas para ambas partes, es decir, con la clasificación del riesgo real, con el número correcto de trabajadores, con los centros de trabajo adecuados y con relaciones laborales dentro de la ley, como por ejemplo las empresas llamadas agrupadoras. Para esta labor la compañía implementó el sistema Avatar, donde las empresas realizan los trámites de afiliación a través de una entrevista, un módulo atendido de manera virtual por un asesor, quien conduce a las nuevas empresas para finalizar con el formulario de afiliación diligenciado. Esto ha permitido frenar afiliaciones irregulares antes de ingresar a los sistemas de la compañía para que después se deban destinar recursos para una reclasificación posterior a la clasificación inicial. Cada visita a una empresa para su reclasificación cuesta $\$ 203.000$.

Tabla 2. Afiliaciones de empresas.

\begin{tabular}{|c|c|c|c|c|}
\hline \multicolumn{5}{|c|}{ Clasificación de empresas inicial } \\
\hline Año & 2013 & 2014 & 2015 & Total \\
\hline $\begin{array}{l}\text { Sin Avatar } \\
\text { (ventanilla) }\end{array}$ & 50.054 & 48.748 & 20.064 & 118.866 \\
\hline Con Avatar & 20.215 & 34.658 & 16.369 & 71.242 \\
\hline $\begin{array}{l}\text { Total } \\
\text { empresas } \\
\text { afiliadas }\end{array}$ & 70.269 & 83.406 & 36.433 & 190.108 \\
\hline$\%$ Avatar & $28,77 \%$ & $41,55 \%$ & $44,93 \%$ & $37,47 \%$ \\
\hline \multicolumn{5}{|c|}{ Fuente: el autor. } \\
\hline \multicolumn{5}{|c|}{$\begin{array}{l}\text { Del total de } 71.242 \text { empresas afiliadas en } 30 \text { meses a } \\
\text { través del aplicativo, fueron reclasificadas } 15.470 \text { y de- } \\
\text { vueltas por información inconsistente } 27.460 \text {, de estas }\end{array}$} \\
\hline \multicolumn{5}{|c|}{ últimas se volvieron a presentar 20.679 empresas con } \\
\hline
\end{tabular}


Tabla 3. Ahorro por la implementación del sistema Avatar.

\begin{tabular}{|c|c|}
\hline Tipo de ahorro & Valor anual \\
\hline Ahorro por no reclasificar empresas & $1.256^{\prime} 164.000$ \\
\hline $\begin{array}{l}\text { Ahorro en futuras reclasificaciones por } \\
\text { devolución de Avatar }\end{array}$ & $2.229^{\prime} 752.000$ \\
\hline Total ahorro & $3.485 ’ 916.000$ \\
\hline
\end{tabular}

Fuente: el autor.

Efecto 2. Reducción en inversiones en programas a través del mapeo de riesgos higiénicos.

- Tipo de efecto: Ahorro en operación

- Cuantificación: \$2.328’048.738.97 por año

Positiva es reconocida por su conocimiento técnico en temas relacionados con promoción y prevención y busca potencializar esta ventaja competitiva y fortalecer los servicios que presta a través del mapeo de riesgos higiénicos (ruido, temperatura y contaminantes químicos) con base en las mediciones ocupacionales realizadas durante el período 2011-2014 y los reportes anuales de datos de vibraciones. Todo esto para brindar soporte técnico e información al proceso de promoción y prevención y orientar la formulación de estrategias masivas que permitan la prevención y control de la exposición a agentes químicos y físicos en las empresas afiliadas; lo anterior busca generar planes masivos de atención a empresas, traduciéndose en una reducción en la inversión en las mediciones de silice, ruido y polvo, así como prevención primaria de la enfermedad laboral asociada a riesgos higiénicos en las empresas objetivo y una menor inversión en planes individuales.

Efecto 3. Desconcentración del riesgo a través del diseño de mecanismo de compensación económico.

- Tipo de efecto: Ingresos efectivos

- C Cuantificación: \$23.222’637.000 para el año 2016
El artículo 76 de la Ley 1753 de 2015 otorgó al gobierno nacional facultades para evitar la concentración de riesgos y la selección adversa en el sistema general de riesgos laborales, indicando que corresponde al ejecutivo definir las "medidas necesarias para evitar la concentración de riesgos y la selección adversa de afiliados por parte de las Administradoras de Riesgos Laborales". A raíz de esto salió el Decreto 2509 de diciembre de 2015, donde se establece en el artículo 1, crear un mecanismo de compensación monetaria con el objeto de corregir los efectos de la concentración del riesgo en el SGRL y sus consecuencias financieras, así como la adopción de medidas para mitigar la problemática.

Para llegar a los resultados obtenidos hubo necesidad de realizar mesas conjuntas donde participaban las compañías aseguradoras, Fasecolda, Ministerio de Hacienda y Crédito Público y el Ministerio del Trabajo, y particularmente Positiva, que conformó un equipo multidisciplinario para esta labor con funcionarios de la Vicepresidencia Técnica, Gerencia de Actuaría, profesionales de gobierno de datos e inteligencia de negocios; también se aplicaron los procedimientos de gobierno de datos para garantizar que la información utilizada para estos cálculos estuviera en las mejores condiciones.

Efecto 4. Optimización del uso de la red asistencial.

- Tipo de efecto: Ahorro por eficiencias

- Cuantificación: \$32.493’000.000.00 por año

Positiva para atender a sus asegurados cuenta con una red de prestadores de servicios de salud, esta red se compone de IPS y centros de rehabilitación los cuales se encargan de atender las necesidades relacionadas con servicios de salud, tales como urgencias, hospitalizaciones, rehabilitaciones y cualquier otro servicio de salud relacionado con las coberturas definidas en los productos en caso de las pólizas de vida o lo definido en la ley para los casos de riesgos laborales. 
Se ha identificado que la compañía necesita ser más eficiente en el manejo de sus recursos, esto con el fin de mejorar los índices de siniestralidad, específicamente en el valor promedio de los siniestros y lograr cumplir la meta corporativa. La aplicación de los componentes del gobierno de datos, ha permitido identificar los posibles puntos en donde se puedan lograr eficiencias, primero, en la negociación de tarifas con la red de IPS y segundo, en el direccionamiento de los afiliados a las IPS negociadas con anterioridad.

\section{CONCLUSIONES}

Se puede afirmar que se han obtenido efectos positivos gracias a la implementación del GD y su integración con el Sistema de Gestión de la Calidad lo cual ha tenido efectos en la operación de la compañía. Los efectos se ven, no solamente a la operación de la compañía, sino también en el Sistema de Gestión de la Calidad, este último ha mejorado gracias a la incorporación de componentes del GD los cuales están alineados con la estrategia corporativa, puntualmente con la meta de lograr a 2018 un índice combinado menor al $100 \%$, dándole fuerza al compromiso de la Dirección. También se definieron protocolos, roles y responsabilidades relacionados con los datos e información, ayudando a entender las interacciones entre procesos. Se mejora considerablemente la administración de la información, gracias a la creación de bodegas de datos oficiales, construcción de herramientas integradoras como CAMI y MAG, se fortalecen los procesos de medición y análisis de métricas y se crea una cultura alrededor de la adecuada gestión de datos e información.

Se crea confianza en las directivas a través de resultados tangibles, esto se puede evidenciar con los resultados de la encuesta de percepción, donde se alcanza un resultado de 3.82 puntos de 5.0 puntos máximos a obtener, teniendo un efecto positivo en las personas y en la cultura organizacional.

A partir de la construcción de los flujos de información, se realizó el levantamiento de los activos de información, esto ha brindado una mirada global a los procesos y permitido que los análisis de eficiencia de procesos con miras a una mejora continua se puedan dar con mayor facilidad al lograr contar con las interacciones claras entre procesos así como de los datos e información que se intercambian.

La cultura de medición en la compañía se ha visto fortalecida gracias a la definición de roles y perfiles de funcionarios que se encarguen de la administración, por lo general las tareas de seguimiento, análisis y reporte son tareas que son impuestas al no contar con directrices claras para la gestión por parte de los líderes de proceso.

Los espacios generados por el proceso de GD son importantes en el sentido de que no se puede consolidar una cultura solamente con un área llevando la carga de la gestión de GD, esa participación activa de los procesos y los usuarios de los datos ha permitido llegar a los resultados mostrados en la encuesta y genera la confianza necesaria para tomar decisiones acertadas en el futuro, soportadas en información confiable.

La implementación de controles en la puerta de entrada son el resultado de los análisis preliminares de las bases de datos en la compañía, esto no hubiese sido posible si no se analizara bajo la perspectiva del GD la información que reposaba en las bases de datos propias, siempre se tratan de implementar estas acciones a manera de proyecto, aplicando los componentes del GD a través de los procedimientos definidos oficialmente en el SIG. Los resultados son contundentes en términos de ahorros por reproceso.

También hay efectos en la sociedad al contar con un mapeo de riesgos higiénicos, esto contribuye en la disminución de la frecuencia y siniestralidad por enfermedades laborales asociadas a riesgos higiénicos en 
el largo plazo, lo cual necesariamente redunda en un beneficio de la sociedad al proveer información que complemente los mapas epidemiológicos del país y mejorar las condiciones de salud de los colombianos.

La participación de proceso del GD en la definición del mecanismo de compensación, fue fundamental, no solamente porque dio orden en la asignación de responsables, sino porque gracias a los ejercicios de calidad de datos, se pudo contar con información confiable que permitiera a su vez correr el modelo matemático que el gobierno nacional dispuso para intervenir el mercado.

Por último, la gestión de optimización de la red asistencial busca brindarles un mejor servicio a los asegurados que sufren un siniestro y quieren ser atendidos de la mejor manera. Los análisis de los históricos de costos de medicamentos, la intervención de la política de distribución de pacientes, la determinación de descentralizar el proceso de negociación en cabeza de los gerentes de la sucursales; todas estas son muestra de los efectos de la aplicación de los componentes del GD en la operación de la compañía.

Analizado el resultado de la presente investigación, así como anteriormente se describen los efectos positivos en la operación también se identificaron algunas falencias que es importante traer a este contexto, de esta misma manera a modo de recomendaciones se presenta un esquema de varios aspectos que deberían tenerse en cuenta para el mantenimiento de los efectos en el tiempo, también se dan respuesta a algunos interrogantes como: ¿Será importante saber cuál es el estado del GD en el sector asegurador?, al respecto se recomienda continuar con el trabajo de investigación en una segunda fase, tratando de ver en el sector asegurador cómo es el desempeño de estas compañías en temas relacionados con la gestión de los datos e información, analizando sus respectivos grados de madurez.
¿La compañía cuenta con el recurso suficiente para atender los temas relacionados con GD? El recurso es insuficiente; se requieren más personas que estén manejando los temas relacionados con GD por el volumen generado al intervenir los problemas de datos en la compañía.

Se recomienda socializar los resultados de los proyectos que tiene componentes del GD a todos los niveles, mejorar los contenidos, el lenguaje; esta información debe estar disponible para todos los funcionarios y se debe articular con la gestión del conocimiento de la compañía, crear un banco de lecciones aprendidas y una red de conocimiento.

Se recomienda implementar una herramienta más robusta de gestión de indicadores (CAMI y MAG), para mejorar el desempeño de los procesos de administración y análisis de datos e información; a la par, se sugiere unificar los repositorios de información, bases de datos y aplicativos, ya que actualmente los usuarios tienen que consultar en varios sitios información que requieren para los análisis y gestión normal de los proceso.

\section{REFERENCIAS}

Al-Ruithe, M., Benkhelifa, E., y Hameed, K. (2016). A conceptual framework for designing data governance for cloud computing. Procedia Computer Science, 94 (Supplement C),160-167.doi:// doi.org.bdatos.usantotomas.edu.co/10.1016/j. procs.2016.08.025

Bibri, S. E., y Krogstie, J. (2017). ICT of the new wave of computing for sustainable urban forms: Their big data and context-aware augmented typologies and design concepts, Sustainable Cities and Society, 32(Supplement C):449-474. doi://doi.org.bdatos. usantotomas.edu.co/10.1016/j.scs.2017.04.012 
Buchmann, M. (2017). The need for competition between decentralized governance approaches for data exchange in smart electricity grids-Fiscal federalism vs. polycentric governance. Journal of Economic Behavior \& Organization, 139, 106-117. doi://doi.org.bdatos.usantotomas.edu. co/10.1016/j.jebo.2017.05.011

Hernández Sampieri, R., Fernández Collado, C., y Baptista Lucio, P. (2014). Metodología de la investigación. México D. F., México: McGraw-Hill.

ICONTEC. (2008). Norma técnica colombiana NTC ISO 9000. Sistema de gestión de la calidad. Fundamentos y vocabulario. Instituto Colombiano de Normas Ténnicas y Certificación. Bogotá D. C., Colombia: ICONTEC.

Mansfield-Devine, S. (2017). Data governance: Going beyond compliance. Computer Fraud \& Security,
(6):12-15. doi://doi.org.bdatos.usantotomas.edu. co/10.1016/S1361-3723(17)30052-0

Seele, P. (2017). Predictive sustainability control: A review assessing the potential to transfer big data driven 'predictive policing' to corporate sustainability management. Journal of Cleaner Production, 153 (Supplement C), 673-686. doi://doi.org.bdatos. usantotomas.edu.co/10.1016/j.jclepro.2016.10.175

Thomas, G. (2009). How to Use the DGI Data Governance Framework to Configure Your Program. Data Governance Institute, 2009. Recuperado de http://www.datagovernance.com/wp-content/ uploads/2014/11/wp_how_to_use_the_dgi_data_ governance_framework.pdf

Thomas, G. (s. f.). The DGI Data Governance Framework. The Data Governance Institute. Recuperado de: http://www.datagovernance.com/wp-content/uploads/2014/11/dgi_framework.pdf. 